\title{
Muscle mass, strength and functional outcomes in critically ill patients after cardiothoracic surgery: does neuromuscular electrical stimulation help? The Catastim 2 randomized controlled trial
}

\author{
Arabella Fischer ${ }^{1 *}$, Matthias Spieg ${ }^{2+}$, Klaus Altmann ${ }^{3+}$, Andreas Winkler ${ }^{4 \dagger}$, Anna Salamon $^{5}$, \\ Michael Themessl-Huber ${ }^{6}$, Mohamed Mouhieddine $^{1}$, Eva Maria Strasser ${ }^{7}$, Arno Schiferer ${ }^{1}$, \\ Tatjana Paternostro-Sluga ${ }^{8}$ and Michael Hiesmayr ${ }^{1}$
}

\begin{abstract}
Background: The effects of neuromuscular electrical stimulation (NMES) in critically ill patients after cardiothoracic surgery are unknown. The objectives were to investigate whether NMES prevents loss of muscle layer thickness $(M L T)$ and strength and to observe the time variation of MLT and strength from preoperative day to hospital discharge.

Methods: In this randomized controlled trial, 54 critically ill patients were randomized into four strata based on the SAPS II score. Patients were blinded to the intervention. In the intervention group, quadriceps muscles were electrically stimulated bilaterally from the first postoperative day until ICU discharge for a maximum of 14 days. In the control group, the electrodes were applied, but no electricity was delivered. The primary outcomes were MLT measured by ultrasonography and muscle strength evaluated with the Medical Research Council (MRC) scale. The secondary functional outcomes were average mobility level, FIM score, Timed Up and Go Test and SF-12 health survey. Additional variables of interest were grip strength and the relation between fluid balance and MLT. Linear mixed models were used to assess the effect of NMES on MLT, MRC score and grip strength.
\end{abstract}

Results: NMES had no significant effect on MLT. Patients in the NMES group regained muscle strength 4.5 times faster than patients in the control group. During the first three postoperative days, there was a positive correlation between change in MLT and cumulative fluid balance $(r=0.43, P=0.01)$. At hospital discharge, all patients regained preoperative levels of muscle strength, but not of MLT. Patients did not regain their preoperative levels of average mobility $(P=0.04)$ and FIM score $(P=0.02)$ at hospital discharge, independent of group allocation.

\footnotetext{
* Correspondence: arabella.fischer@meduniwien.ac.at

${ }^{\dagger}$ Equal contributors

'Division of Cardiothoracic and Vascular Anesthesia and Intensive Care

Medicine, Department of Anesthesia, General Intensive Care and Pain

Control, Medical University of Vienna, Währingergürtel 18-20, 1090 Vienna,

Austria

Full list of author information is available at the end of the article
} 
(Continued from previous page)

Conclusions: NMES had no effect on MLT, but was associated with a higher rate in regaining muscle strength during the ICU stay. Regression of intramuscular edema during the ICU stay interfered with measurement of changes in MLT. At hospital discharge patients had regained preoperative levels of muscle strength, but still showed residual functional disability and decreased MLT compared to pre-ICU levels in both groups.

Trial registration: Clinicaltrials.gov identifier NCT02391103. Registered on 7 March 2015.

Keywords: Electrical stimulation therapy, ICU-acquired weakness, Muscular atrophy, Ultrasonography, Muscle layer thickness, Edema, Muscle strength, Critically ill, Cardiothoracic surgery, Functional outcomes

\section{Background}

Intensive care unit-acquired weakness (ICUAW) affects 24-77\% of patients with an ICU stay longer than 1 week [1]. The main risk factors for ICUAW development include sepsis, catecholamine administration, hyperglycemia, ICU length of stay and immobility [1]. ICUAW is associated with symptomatic aspirations [2] and increased hospital mortality [1].

At 5 years after ICU discharge, the 6-minute walking distance (6MWD) and the quality of life assessed by the 36-Item Short Form (SF-36) questionnaire are still decreased [3]. Activities in daily living and cognitive function are impaired even up to 8 years after ICU discharge [4]. In order to prevent these tremendous consequences of ICUAW, early diagnosis and treatment may be decisive. In nonsedated patients, ICUAW is diagnosed when Medical Research Council (MRC) sum score is below 48 out of 60 points or when mean MRC score is below 4 out of 5 points in all testable muscle groups [5]. In sedated patients, muscle ultrasonography may be a diagnostic tool to detect muscle wasting [6, 7]. It is unclear whether muscular ultrasonography is influenced by edema [6-13]. Among different treatment modalities of ICUAW [14, 15], neuromuscular electrical stimulation (NMES) is a feasible therapy for neuromuscular activation in sedated patients. Yet, the effects of NMES on muscle mass and strength are still unclear in critically ill patients: randomized controlled trials either showed no effect $[11,16,17]$ or (partially) beneficial effects [9, 17-22]. Moreover, most prior NMES studies did not assess any functional outcomes at hospital discharge to assess the potential effect of NMES on the patient's functional status after the ICU stay $[9,11,17,19-21]$. NMES can be safely applied in critically ill patients with external pacemakers after cardiothoracic surgery [23, 24].

To date, no randomized controlled trial has evaluated the effects of NMES on muscle mass and strength in a cardiothoracic surgery patient population. Given that cardiovascular disease is the leading cause of death worldwide [25], more attention needs to be addressed to the rehabilitation of critically ill patients after cardiac surgery. Neither have trials assessed muscle mass, strength and functional status altogether prior to critical illness, which is necessary for evaluating change in muscle mass and strength in relation to preoperative values.

Therefore the first objective was to investigate whether early NMES is effective in preventing loss of MLT and strength in critically ill patients after cardiothoracic surgery. The second objective was to observe the time variation of muscle layer thickness (MLT) and muscle strength from preoperative day to hospital discharge. To adjust for possible confounders for measurement of MLT, daily fluid balance was assessed during the ICU stay.

\section{Methods}

\section{Study design and population}

The prospective, randomized, controlled, single-blind trial Catastim 2 was carried out at the Division of Cardiothoracic and Vascular Anesthesia and Intensive Care Medicine of the General Hospital affiliated to the Medical University of Vienna from May 2011 to July 2012 (clinicaltrials.gov identifier: NCT02391103). Patients were blinded to the NMES/sham stimulation. Nonblinded assessors performed the ultrasound scans; however, the evaluators measuring MLT were blinded to patients' group assignment. The assessors of muscle strength and the secondary outcomes were not blinded to patients' group assignment. Ethical approval was obtained from the ethics committee of the Medical University of Vienna in 2010 (number 1072/ 2010). Patients were included if they underwent cardiothoracic surgery and were anticipated to stay in the ICU for at least 48 hours. Patients were excluded if they were younger than 18 years, had a body mass index of more than $40 \mathrm{~kg} / \mathrm{m}^{2}$, had metal implants or skin lesions in the stimulation area, had neuromuscular diseases, had an implanted ventricular assist device or intra-aortic balloon pump. After submission of a protocol amendment in June 2011, a twofold study setting was defined in order to recruit patients not only before, but also after cardiothoracic surgery. In cohort A, patients were recruited before surgery. In cohort $\mathrm{B}$, patients were recruited after surgery. Recruitment of patients after surgery primarily allowed inclusion of patients after urgent nonelective surgeries such as heart transplantations, who could not be seen preoperatively. Written informed consent was obtained from 
patients in cohort A. Patients in cohort B were included in the trial while sedated to be later informed of their participation. The inclusion of sedated patients in cohort B without providing written informed consent was approved by the ethics committee of the Medical University of Vienna.

\section{Randomization}

For all patients, randomization was performed on postoperative day 1. Patients in cohort A and B were separately randomized using a web-based randomizer for clinical trials [26]. In the previous Catastim 1 trial taking place in the same ICU [24], the NMES and control groups had slightly different Simplified Acute Physiology Score II (SAPS II) scores. Hence, in the Catastim 2 trial, randomization was stratified by the SAPS II score on the first postoperative day to ensure balance of the NMES and control groups with respect to disease severity. The four strata were deduced from the quartiles of SAPS II scores in a retrospective analysis of 2708 patients of the same ICU.

\section{Intervention}

In the intervention group, all parts of the quadriceps muscle (rectus femoris muscle, vastus intermedius muscle, vastus lateralis muscle, vastus medialis muscle) of both thighs were electrically stimulated. The muscles were stimulated twice a day $(2 \times 30$ minutes of NMES with an interval of at least 30 minutes between both sessions [24]) 7 days a week during the entire ICU stay but no longer than 14 days, starting on postoperative day 1 . Highest tolerable intensity just below the pain threshold was applied. A visible and palpable contraction was the goal to achieve. The Compex 3 Professional (CefarCompex Medical AB, Freiburg, Germany) stimulator delivered biphasic rectangular pulses of $0.4 \mathrm{~ms}$ pulse duration at $66 \mathrm{~Hz}$ [19]. The duty cycle was $3.5 \mathrm{~s}$ on and $4.5 \mathrm{~s}$ off, ramping up and down were both set at $0.5 \mathrm{~s}$ [24]. Dual Snap $(5 \times 10 \mathrm{~cm}$ and $5 \times 5 \mathrm{~cm}$ ) electrodes (CefarCompex Medical AB) were used. In the control group, the electrodes were applied, connected to the stimulator, but no electricity was delivered. Patients were blinded to the intervention: All patients were told they would get NMES, whether or not electricity was delivered.

\section{Outcomes}

\section{Muscle layer thickness (MLT)}

For all patients, MLT of the quadriceps muscle of both thighs using two-dimensional B-mode ultrasound (Vivid i, General Electric Healthcare, Little Chalfont, UK) was measured on postoperative day 1 , every other day until ICU discharge and at hospital discharge. Patients in cohort A had an additional assessment of MLT before surgery. In cohort A, the preoperative assessment took place on the preoperative day in most patients $(n=23)$. Due to postponement of surgery, the preoperative assessment was between 2 and 8 days before surgery in six patients. In previous studies $[7,27,28]$ the cross-sectional area of the rectus femoris muscle was measured. However, the rectus femoris muscle only constitutes approximately $10 \%$ of the total quadriceps cross-sectional area [29]. Even though there is no data about differing wasting rates of the quadriceps muscle components in critically ill patients, hypertrophy may occur at different rates in the quadriceps muscle components in healthy young males during training [30]. Thus we measured not a crosssectional area of a single muscle, but the muscle layer thickness of all parts of the quadriceps muscle. During the ICU stay, edema may enlarge the cross-sectional area of the muscle. Furthermore the ultrasound transducer is only $3.8 \mathrm{~cm}$ wide. Thus it is impossible to measure all parts of the quadriceps muscle at only one measuring point by ultrasonography. In order to account for all parts of the quadriceps muscle in the most reliable way, we used three measuring points per leg in two orthogonal planes to increase precision. Twelve measurements per patient per observation day were averaged to account for all parts of the quadriceps muscle. Three measuring points per leg were marked with a permanent marker pen for scanning in the transverse and sagittal plane to make sure that the exact same measuring point was measured across time [31]: (1) lateral point: in the middle of the distance between the superior border of the greater trochanter and the lateral knee joint space; (2) ventral point: on a line from the anterior superior iliac spine to the superior border of the patellar base, on the same level as the lateral point; (3) medial point: on a line from the pubis to the medial knee joint space, on the same level as the lateral point. Frequency was set at $6 \mathrm{MHz}$. A pillow was placed under the patient's knees to ensure muscular relaxation. A gel pad (Geliperm $260 \times 120 \times 3.3 \mathrm{~mm}$, Geistlich Pharma, Wolhusen, Switzerland) placed on the patient's thigh and additional ultrasound gel was used to apply the least amount of pressure necessary while scanning until the muscles were neatly displayed. Prior to trial start, interexaminer variation in MLT scanning between the five trained assessors was assessed on 24 measuring points. The intra-class correlation coefficient (ICC) for the five assessors was 0.9 (95 \% CI, 0.82 to 0.95$)(P<0.001)$. The five nonblinded assessors effectuated the ultrasound scans and muscle strength assessment and then applied the electrodes for NMES or sham stimulation. During the recruitment phase, none of the nonblinded assessors had access to any actual MLT values. Thus it appears unlikely that any observer bias was introduced by the nonblinded assessors who effectuated the ultrasound scans. After the end of data collection, MLT was evaluated with the software EchoPAC (General Electric Healthcare). The two evaluators were blinded to whether the patient belonged to the 
NMES or control group. Scans were evaluated per patient from the first to the last taken ultrasound scan to ensure consistency since muscular form is very individual. In that way, the level of the fascia lata could be discerned consistently in each patient. In a pilot testing we observed that the intramuscular fascia cannot be precisely delimitated from the muscular parts in patients during early ICU stay (Fig. 1 and Additional file 1). Thus we opted to measure the entire muscle layer thickness from the fascia lata (or deep fascia) to the femur, because these two landmarks can be precisely delineated. Beginning in the exact center of the scan at the level of the lowest layer of the fascia lata, MLT was measured down to the femur drawing the shortest possible line. At the lateral measuring point in the transverse and sagittal plane, MLT of vastus lateralis and vastus intermedius muscles was measured from the lowest layer of the fascia lata to the femur (Fig. 1 and Additional file 1). Similarly, MLT of rectus femoris and vastus intermedius muscles was measured in both planes at the ventral measuring point and MLT of vastus medialis and vastus intermedius muscles at the medial measuring point. MLT measurement in both the sagittal and transverse plane of one measuring point is shown in Additional file 1. MLT measured in the transverse plane highly correlated with the corresponding MLT measured in the sagittal plane $(r=0.97, P<0.001)$. We averaged the tranverse and sagittal measurements for each measuring point. This accounts for interscan variation per measuring point. Since preliminary results indicated that the mean of all six measuring points in both planes is representative of all 12 measurements, mean MLT of all 12 measurements was calculated for each observation day.

\section{Muscle strength}

For all patients, muscle strength was measured on postoperative day 1 or as soon as they were awake, every day until ICU discharge and at hospital discharge. The patient was considered awake when he could follow orders, which is necessary for evaluation of active muscle contractions. Patients in cohort A had an additional assessment of muscle strength before surgery. Based on the diagnostic criteria of ICUAW, which is established when mean MRC score of all testable muscle groups is below 4 [5], muscle strength was evaluated in all muscle groups of the upper and lower extremities using the MRC scale [32]. Mean MRC score of all muscle groups was calculated [5]. In addition quadriceps mean MRC score was calculated as mean MRC score of knee extension and hip flexion of both legs to evaluate the function of the electrically stimulated quadriceps muscles. According to the MRC scale [33], mean MRC score ranges from a minimum of 0 to a maximum of 5 points. Bilateral grip strength was measured with a Jamar hand dynamometer (Jamar, Duluth, MN, USA). Patients were asked to perform the hand dynamometer test twice for each side and the highest value was noted for each side.

\section{Functional outcomes}

For all patients, the Functional Independence Measure (FIM) score [34], the Timed Up and Go test [35] and the 12-Item Short Form (SF-12) health survey [36] were evaluated at hospital discharge. The average mobility level proposed by Brown [37] was assessed at ICU and hospital discharges. Patients in cohort A had an additional assessment of all functional outcomes before surgery.

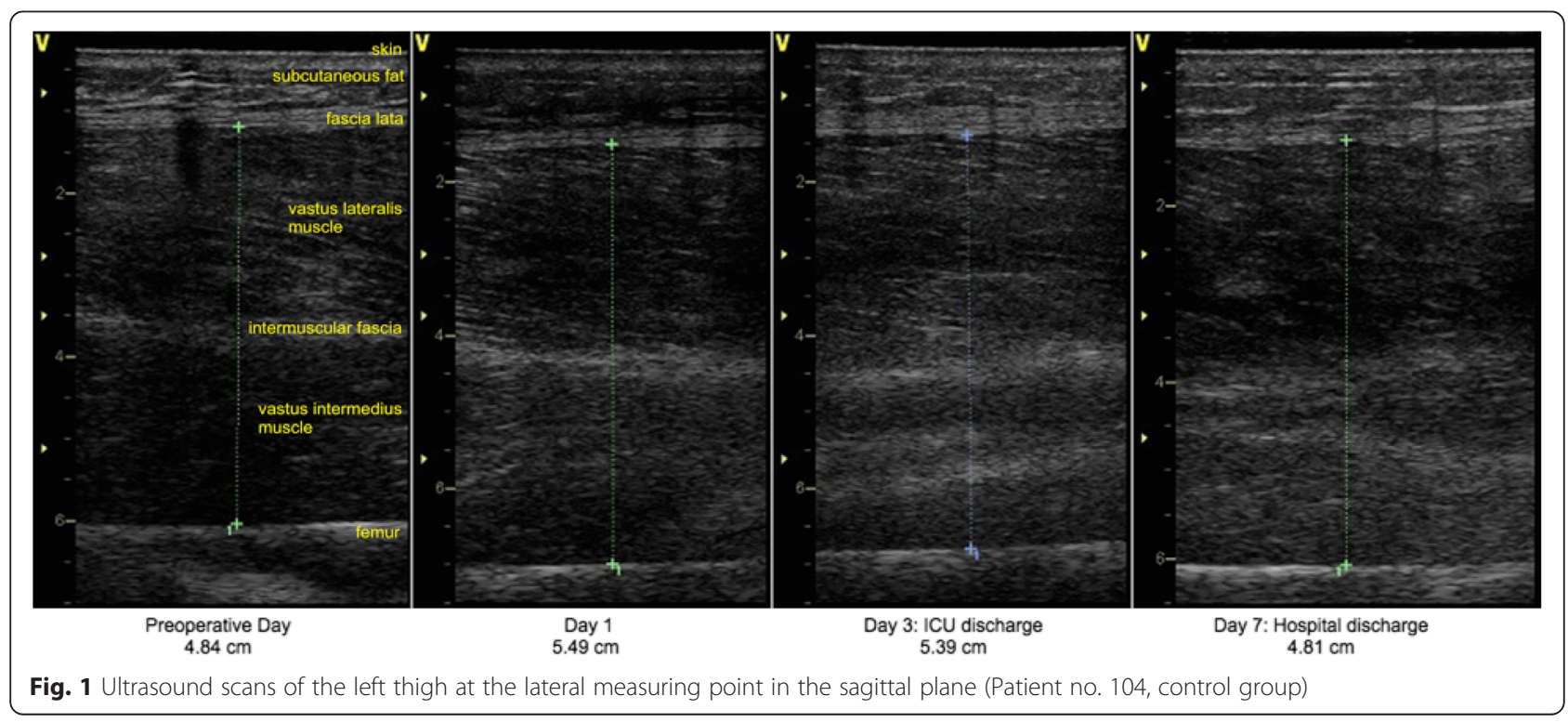




\section{Patient satisfaction}

At ICU discharge, patients were asked if the NMES/ sham stimulation was comfortable or uncomfortable.

\section{Statistical analysis}

In the previous Catastim 1 trial [24], MRC muscle strength was evaluated for hip flexion: mean changes in MRC score from the first postoperative day until ICU discharge were -0.80 points [standard deviation (SD), 0.70 points] in the NMES group $(n=20)$ versus -1.75 points (SD, 1.52 points) in the control group out of a maximum MRC score of 5 points $(n=20)(P=0.015)$. Based on these results, sample size calculation with Stata yielded 25 patients per group at a two-sided significance level of $\alpha=0.05$ and $80 \%$ power. Assuming a dropout rate of $20 \%, 60$ patients needed to be randomized.

All data was analyzed according to the intention-totreat principle with no imputation for any missing data. Normality was assessed with Kolmogorov-Smirnov and Shapiro-Wilk statistics. If quantitative variables were normally distributed, they were expressed as mean \pm SD or $95 \%$ confidence interval $(\mathrm{CI})$. If they were not normally distributed, median (range) was indicated.

In previous muscle ultrasound studies, linear regression analyses were performed [7, 27, 28]. Similarly, we presented results of actual raw data (Additional file 2 and Table 7) and results based on linear regression modeling (Tables 2, 3, 4, 5, 6 and Additional file 3). Linear mixed models were used to assess the treatment effects while accounting for the repeated measurements per patient. Thus, the random effect 'patient' was defined in each model. Linear mixed models were calculated for mean MLT, mean MRC score of all muscle groups, quadriceps mean MRC score and grip strength. Fixed effects of interest were postoperative day or study day, NMES/control group, interaction between NMES/control group and postoperative day, and daily fluid balance in the ICU. Fixed effects were either considered as quantitative or categorical variables: for example, the postoperative day or the daily fluid balance at the ICU were quantitative variables. The study day (preoperative day, first postoperative day, ICU discharge, hospital discharge) or the group (NMES group, control group) were categorical variables. By definition the results presented as linear mixed models are derived statistics by linear regression modeling ('proc mixed' in SAS software version 9.4; SAS Institute Inc., Cary, NC, USA).

Because of bandages to protect arterial catheters, patients were not always able to perform every movement in all 12 muscle groups. If there were missing values for muscle groups, mean MRC score was calculated for the muscle groups that could be assessed. Similarly, due to armboards because of radial arterial catheter, patients were not always able to perform the hand dynamometer test on both sides. Thus, either the right or left grip strength was chosen for statistical analysis per patient according to the lowest number of missing values over all study days on each side. If the number of missing values was equal for right and left grip strength for a patient, either the right or left grip strength was chosen according to the highest mean grip strength over all study days on each side.

In addition, intraoperative fluid balance, changes over time in MLT or mean MRC score were compared between both groups with independent-groups $t$ tests or Mann-Whitney $U$ tests as appropriate. Change in MLT was correlated with the cumulative fluid balance in the first three postoperative days with Pearson productmoment correlation.

The secondary functional outcomes were evaluated in a sensitivity analysis for patients seen both on preoperative day and at hospital discharge $(n=12)$ in order to evaluate whether preoperative functional levels could be regained at hospital discharge. Changes over time in secondary functional outcomes were analyzed with Wilcoxon signed-rank tests. Changes in functional outcomes were compared between both groups with Mann-Whitney $U$ tests. The patient satisfaction related to the intervention was evaluated in 42 patients with Pearson's chi-squared test or Fisher's exact test as appropriate.

Significance level was set at 0.05 . All $P$ values were twotailed. For statistical analysis, SPSS version 22 (IBM Corp., Armonk, NY, USA) and SAS version 9.4 were used. For figure construction, GraphPad Prism version 6.0 (GraphPad Software, Inc., La Jolla, CA, USA) was used.

\section{Results}

Patients

Fifty-four patients were included in the intention-totreat analysis: 27 belonged to the NMES group and 27 to the control group (Fig. 2). All baseline characteristics were comparable in both groups except for dobutamine and noradrenaline doses in the ICU (Table 1).

\section{NMES}

Median duration of the electrical or sham stimulation was 4 days (range, 2-13 days) in the NMES group and 4 days (range, 1-14 days) in the control group. In the NMES group, median NMES intensities were $40.5 \mathrm{~mA}$ (range, 2-100 $\mathrm{mA}$ ) for the right thigh and $40 \mathrm{~mA}$ (range, $5-120 \mathrm{~mA}$ ) for the left thigh. In terms of adherence, only 9 out of 145 NMES sessions and 6 out of 154 sham stimulation sessions could not have been carried out.

\section{Muscle layer thickness (MLT)}

MLT was evaluated in 53 out of 54 patients. Due to technical reasons, ultrasound scans of one patient could not be saved. MLT was not evaluated in 210 of all 2825 


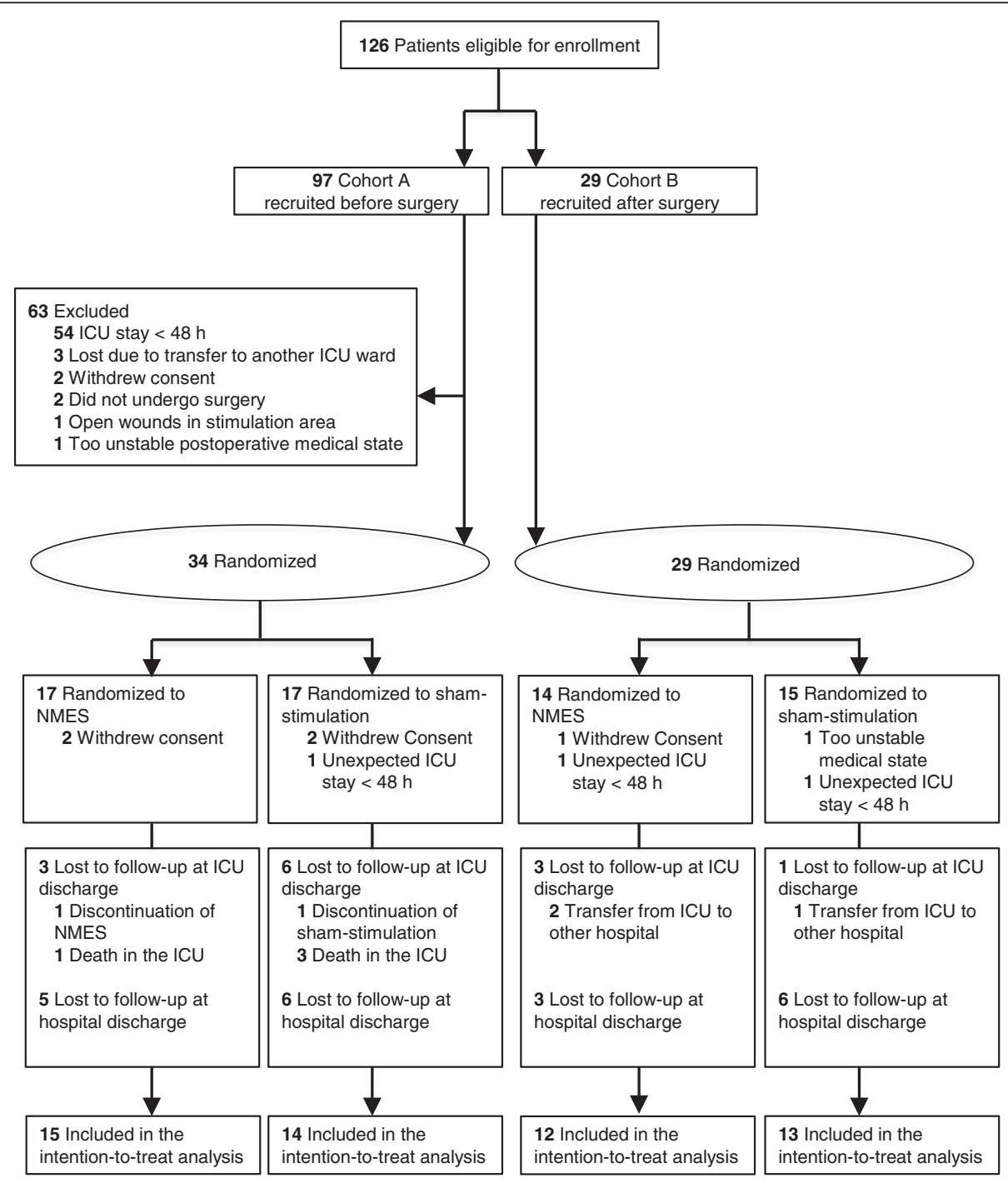

Fig. 2 Flow diagram of the Catastim 2 trial

ultrasound scans, because the muscles of the thigh could not be clearly delimited (Additional file 4). MLT increased from preoperative day to the first postoperative day by $0.41 \mathrm{~cm}(95 \% \mathrm{CI}$ of estimate, 0.24 to $0.59 \mathrm{~cm})$ $(P<0.001)$ (Table 3). Intraoperative fluid balance was $4856.5 \pm 2637.4 \mathrm{ml}($ mean $\pm \mathrm{SD})$ in the NMES group $(n=26)$ and $4111.9 \pm 1766.6 \mathrm{ml}($ mean $\pm \mathrm{SD})$ in the control group $(n=26)(P=0.24)$. During the first three postoperative days, there was a positive correlation between change in MLT and cumulative fluid balance $(r=0.43, P=0.01)(n=41)$. From the first postoperative day, MLT decreased by $0.08 \mathrm{~cm}(95 \% \mathrm{CI}$ of estimate, -0.11 to $-0.06 \mathrm{~cm})$ per day $(P<0.001)$. The change in MLT of $-0.08 \mathrm{~cm}$ per day is the result of the linear regression from the first postoperative day where each patient is considered as a random factor. Overall this means that MLT decreases by $8 \mathrm{~mm}(95 \% \mathrm{CI},-11$ to -6 $\mathrm{mm}$ ) in 10 days by linear regression modeling (Table 2). This corresponds to a $25 \%$ decrease in muscle layer thickness in 10 days. The NMES intervention had no significant effect on MLT (Table 2). At ICU discharge, MLT was about $0.18 \mathrm{~cm}$ (95\% CI of estimate, -0.004 to $0.37 \mathrm{~cm})$ higher than on preoperative day $(P=0.055)$ (Table 3). At hospital discharge, MLT was about 0.28 $\mathrm{cm}(95 \% \mathrm{CI}$ of estimate, -0.49 to $-0.06 \mathrm{~cm})$ lower than on preoperative day $(P=0.01)$ (Table 3$)$. Time variation of actual MLT values in individual patients is shown in Additional file 2.

\section{Muscle strength}

Out of all 54 patients, 36 patients were awake on postoperative day 1,7 patients on postoperative day 2 or 3,8 patients on postoperative days 4 to 7 in order to perform the first postoperative MRC measurement in the ICU. 
Table 1 Baseline characteristics of patients

\begin{tabular}{|c|c|c|}
\hline & $\begin{array}{l}\text { NMES group } \\
(n=27)\end{array}$ & $\begin{array}{l}\text { Control group } \\
(n=27)\end{array}$ \\
\hline Age, mean (SD), y & $63.3(15.5)$ & $69.7(13.1)$ \\
\hline Male sex, no. (\%) & 18 & 20 \\
\hline Body mass index, mean (SD) & $27.6(3.7)$ & $27.7(4.6)$ \\
\hline $\begin{array}{l}\text { SAPS II at postoperative day } 1, \\
\text { median (range) }\end{array}$ & $26.0(7-46)$ & $24.0(7-47)$ \\
\hline $\begin{array}{l}\text { SOFA at postoperative day } 1, \\
\text { median (range) }\end{array}$ & $9.0(1-15)$ & $7.0(1-11)$ \\
\hline $\begin{array}{l}\text { Period ventilated, median } \\
\text { (range), } \mathrm{d}^{a}\end{array}$ & $2(1-7)$ & $2(1-15)$ \\
\hline $\begin{array}{l}\text { ICU length of stay, median } \\
\text { (range), } d^{b}\end{array}$ & $6(3-23)^{c}$ & $7(3-213)^{c}$ \\
\hline ICU readmission, no. (\%) & $4(14.8)$ & $3(11.1)$ \\
\hline $\begin{array}{l}\text { ICU readmission, median } \\
\text { (range), } d^{b}\end{array}$ & $2(1-35)$ & $6(1-41)$ \\
\hline $\begin{array}{l}\text { Hospital length of stay, } \\
\text { median (range), } d^{b}\end{array}$ & $22(4-84)^{d}$ & $19(9-213)^{d}$ \\
\hline \multicolumn{3}{|l|}{ Mortality, no (\%) } \\
\hline in the ICU & $1(3.7)$ & $3(11.1)$ \\
\hline at the surgical ward & 0 & 0 \\
\hline Single surgical procedure, no. $(\%)^{e}$ & $17(63.0)$ & $17(63.0)$ \\
\hline Double surgical procedure, no. $(\%)^{e}$ & $7(25.9)$ & $7(25.9)$ \\
\hline Triple surgical procedure, no. $(\%)^{e}$ & $3(11.1)$ & $3(11.1)$ \\
\hline \multicolumn{3}{|l|}{$\begin{array}{l}\text { Surgery type, no. of procedures (\% } \\
\text { in relation to total no. of procedures) }\end{array}$} \\
\hline Aortic valve replacement & $9(22.5)$ & $11(27.5)$ \\
\hline Coronary artery bypass grafting & $11(27.5)$ & $8(20)$ \\
\hline Heart transplantation & $6(15)$ & $5(12.5)$ \\
\hline $\begin{array}{l}\text { Other cardiothoracic } \\
\text { surgery type }\end{array}$ & $4(10)$ & $4(10)$ \\
\hline Mitral valve replacement & $2(5)$ & $5(12.5)$ \\
\hline Mitral valve reconstruction & $4(10)$ & $3(7.5)$ \\
\hline Tricuspid valve reconstruction & $3(7.5)$ & $2(5)$ \\
\hline Bentall procedure & $1(2.5)$ & $2(5)$ \\
\hline \multicolumn{3}{|l|}{$\begin{array}{l}\text { Comorbidities present in } 96.3 \% \text { of the } \\
\text { study population, no. (\%) }\end{array}$} \\
\hline Coronary heart disease & $12(44.4)$ & $8(29.6)$ \\
\hline Hypertension & $9(33.3)$ & $7(25.9)$ \\
\hline Myocardial infarction & $7(25.9)$ & $4(14.8)$ \\
\hline Atrial fibrillation & $2(7.4)$ & $8(29.6)$ \\
\hline Diabetes & $5(18.5)$ & $4(14.8)$ \\
\hline Obesity & $3(11.1)$ & $6(22.2)$ \\
\hline $\begin{array}{l}\text { Chronic obstructive } \\
\text { pulmonary disease }\end{array}$ & $6(22.2)$ & $3(11.1)$ \\
\hline Hyperlipidemia & $4(14.8)$ & $4(14.8)$ \\
\hline Malignoma & $2(7.4)$ & $5(18.5)$ \\
\hline Carotid artery stenosis & $2(7.4)$ & $2(7.4)$ \\
\hline
\end{tabular}

Table 1 Baseline characteristics of patients (Continued)

Patients receiving medication in the ICU, no. (\%)

$\begin{array}{lll}\text { Opioids } & 26(96.3) & 26(96.3) \\ \text { Insulin } & 25(92.6) & 26(96.3) \\ \text { Dobutamine } & 21(77.8) & 17(63) \\ \text { Noradrenaline } & 18(66.7) & 18(66.7) \\ \text { Glucocorticoids } & 11(40.7) & 6(22.2) \\ \text { Benzodiazepines } & 6(22.2) & 6(22.2) \\ \text { Neuromuscular blockers } & 4(14.8) & 2(7.4) \\ \text { Itake days, median (range), d } & & \\ \text { Opioids } & 3(1-10) & 3(1-14) \\ \text { Insulin } & 4(1-9) & 4(1-14) \\ \text { Dobutamine } & 4(2-12) & 5(1-14) \\ \text { Noradrenaline } & 2(1-9) & 4.5(1-14) \\ \text { Glucocorticoids } & 3(1-7) & 3.5(1-14) \\ \text { Benzodiazepines } & 1(1-4) & 1(1-9) \\ \text { Neuromuscular blockers } & 1(1-1) & 1(1-1)\end{array}$

Dose, median (range), $\mu \mathrm{g} / \mathrm{kg} / \mathrm{min}$

$\begin{array}{lll}\text { Dobutamine } & 2.78(0.52-7.59)^{9} & 4.12(0.82-10.53)^{g} \\ \text { Noradrenaline } & 0.07(0.01-0.54)^{h} & 0.12(0.002-0.88)^{h}\end{array}$

NMES neuromuscular electrical stimulation, SD standard deviation, SAPS II Simplified Acute Physiology Score II, SOFA Sequential Organ Failure Assessment, ICU intensive care unit

${ }^{a}$ The ventilation period was calculated as the number of calendar days from the day of surgery (counted as 1 day) to the last day of ventilation (also counted as 1 day). All patients were ventilated when transferred from the operating room to the ICU

${ }^{b}$ The time period was calculated as the number of calendar days from the day of ICU/hospital (re)admission (counted as 1 day) to the day of ICU/hospital discharge (also counted as 1 day unless discharged on the same day as the (re)admission to ICU)

${ }^{c} P=0.46$

${ }^{\mathrm{d}} P=0.60$

${ }^{e}$ Example of single surgical procedure: aortic valve replacement; example of double surgical procedure: aortic valve replacement + coronary artery bypass grafting; example of triple surgical procedure: aortic valve replacement + mitral valve reconstruction + coronary artery bypass grafting

${ }^{f}$ Atrial septal defect II closure, pericardial tamponade, lung transplantation, resection of cardiac aneurysm, aortic arch replacement, Glenn procedure, Ross procedure, replacement of ascending aorta ${ }^{g} P<0.001$

${ }^{\mathrm{h}} P=0.01$

Three patients remained sedated during the first 14 postoperative days and were not able to undergo MRC evaluation during their ICU stay. All patients in cohort A, who were assessed before surgery, had normal muscle strength (mean MRC score of 4.92 points (3.83 to 5 points), median (range), in the NMES group versus 4.92 points (3.67 to 5 points), median (range), in the control group) ( $P$ $=0.96$ ). Mean MRC score of all muscle groups decreased from preoperative day to the first postoperative day by 0.57 points ( $95 \% \mathrm{CI}$ of estimate, -0.78 to -0.36 points) $(P<$ 0.001) (Table 5). The decrease in mean MRC score of all muscle groups from preoperative day to postoperative day 1 was significantly higher in the NMES group [median (range): -0.65 points $(-1.82$ to 0 points)] than in the 
Table 2 Linear mixed model for MLT from the first postoperative day for a maximum of 14 postoperative days (53 patients, 183 observations)

\begin{tabular}{lll}
\hline & $\begin{array}{l}\text { Estimate of MLT in } \\
\text { cm }(95 \% \mathrm{Cl})\end{array}$ & $P$ value \\
\hline Intercept & $3.87(3.58$ to 4.15$)$ & $<0.001$ \\
Postoperative day & $-0.08(-0.11$ to -0.06$)$ & $<0.001$ \\
Control group & Reference & $\cdot$ \\
NMES group & $-0.18(-0.59$ to 0.23$)$ & 0.38 \\
Postoperative day $\times$ Control group & Reference & $\cdot$ \\
Postoperative day $\times$ NMES group & $0.02(-0.01$ to 0.06$)$ & 0.21 \\
Daily fluid balance in the ICU & $-0.04(-0.08$ to 0.01$)$ & 0.09 \\
\hline
\end{tabular}

$M L T$ muscle layer thickness, $\mathrm{Cl}$ confidence interval, NMES neuromuscular electrical stimulation, ICU intensive care unit

control group [median (range): -0.11 points ( -2.36 to 0.18 points)] $(P=0.046)$. Thus, even before the NMES intervention began, patients randomized to the NMES group had a weaker mean MRC of -0.45 points $(95 \% \mathrm{CI}$ of estimate, -0.88 to -0.03 points, $P=0.04$ ) than patients randomized to the control group at the starting point, which was the first postoperative day (Table 4). This is unrelated to the NMES intervention because the intervention started only afterwards. After the onset of the NMES intervention, patients in the NMES group had a 4.5 times higher slope in recovering muscle strength than patients in the control group by linear regression modeling [0.09 MRC points per day ( $95 \% \mathrm{CI}$ of estimate, 0.03 to 0.14 MRC points per day) in the NMES group versus 0.02 MRC points per day ( $95 \%$ $\mathrm{CI}$ of estimate, -0.02 to $0.05 \mathrm{MRC}$ points per day) in the control group] (Table 4). Finally, mean MRC score of all muscle groups regained preoperative values at hospital discharge independent of group allocation $(P=0.43)$ (Table 5). Time variation of actual mean MRC score of all muscle groups in individual patients is shown in Additional file 2. Analysis of quadriceps mean MRC showed similar results to those of mean MRC of all muscle groups (Additional file 3). Patients had lost 4 kilogram-force (95\% CI of estimate, -6.90 to -1.09 kilogram-force) in hand grip

Table 3 Linear mixed model for MLT on four important study days (53 patients, 141 observations)

\begin{tabular}{lll}
\hline & Estimate of MLT in cm (95 \% Cl) & $P$ value \\
\hline Intercept & $3.26(2.99$ to 3.52$)$ & $<0.001$ \\
Preoperative day & Reference & $\cdot$ \\
First postoperative day & $0.41(0.24$ to 0.59$)$ & $<0.001$ \\
ICU discharge & $0.18(-0.004$ to 0.37$)$ & 0.055 \\
Hospital discharge & $-0.28(-0.49$ to -0.06$)$ & 0.01 \\
Control group & Reference & $\cdot$ \\
NMES group & $-0.16(-0.49$ to 0.16$)$ & 0.32 \\
\hline
\end{tabular}

$M L T$ muscle layer thickness, $\mathrm{Cl}$ confidence interval, $I C U$ intensive care unit, NMES neuromuscular electrical stimulation
Table 4 Linear mixed model for mean MRC of all muscle groups ${ }^{\mathrm{a}}$ from the first postoperative day for a maximum of 14 postoperative days (51 patients, 220 observations)

\begin{tabular}{lll}
\hline & $\begin{array}{l}\text { Estimate of mean MRC } \\
\text { in points }{ }^{\mathrm{a}}(95 \% \mathrm{Cl})\end{array}$ & $P$ value \\
\hline Intercept & $4.10(3.80$ to 4.39$)$ & $<0.001$ \\
Postoperative day & $0.02(-0.02$ to 0.05$)$ & 0.40 \\
Control group & Reference & $\cdot$ \\
NMES group & $-0.45(-0.88$ to -0.03$)$ & 0.04 \\
Postoperative day $\times$ Control group & Reference & $\cdot$ \\
Postoperative day $\times$ NMES group & $0.09(0.03$ to 0.14$)$ & 0.002 \\
\hline
\end{tabular}

Days of ICU and hospital discharge, where no NMES was applied anymore, were excluded in this model

The linear mixed model for mean MRC in Table 4 reads as follows: $M R C=4.10+(0.02 \times$ postoperative day $)-(0.45 \times$ NMES group $)+(0.09 \times$ postoperative day $\times$ NMES group)

0.02 is the slope of MRC time variation in the control group, which is the reference group: for each postoperative day, MRC increases by 0.02 points ( $95 \% \mathrm{Cl},-0.02$ to 0.05 points) in the control group $(P=0.40)$ 0.45 represents the lower starting point in the NMES group on the first postoperative day before the NMES intervention began: on the first postoperative day, MRC was about -0.45 points $(95 \% \mathrm{Cl},-0.88$ to -0.03 points) lower in the NMES group than in the control group $(P=0.04)$ 0.09 is the slope of MRC time variation in the NMES group: the slope of MRC time variation is 4.5 times higher than the slope in the control group $(P=0.002)$

MRC Medical Research Council, Cl confidence interval, NMES neuromuscular electrical stimulation

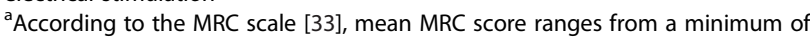
0 to a maximum of 5 points

Table 5 Linear mixed model for mean MRC of all muscle groups on four important study days (51 patients, 130 observations)

\begin{tabular}{lll}
\hline & $\begin{array}{l}\text { Estimate of mean MRC } \\
\text { in points }{ }^{\mathrm{a}}(95 \% \mathrm{Cl})\end{array}$ & $P$ value \\
\hline Intercept & $4.69(4.45$ to 4.93$)$ & $<0.001$ \\
Preoperative day & Reference & $\cdot$ \\
First postoperative day & $-0.57(-0.78$ to -0.36$)$ & $<0.001$ \\
ICU discharge & $-0.27(-0.48$ to -0.06$)$ & 0.01 \\
Hospital discharge & $0.10(-0.15$ to 0.35$)$ & 0.43 \\
Control group & Reference & $\cdot$ \\
NMES group & $0.01(-0.26$ to 0.28$)$ & 0.92
\end{tabular}

The linear mixed model for mean MRC in Table 5 reads as follows: On the first postoperative day mean MRC was about -0.57 points $(95 \% \mathrm{Cl}$, -0.78 to -0.36 points) lower than on preoperative day, which is the reference day $(P<0.001)$

At ICU discharge mean MRC was about -0.27 points $(95 \% \mathrm{Cl},(-0.48$ to -0.06 points) lower than on preoperative day $(P=0.01)$

At hospital discharge mean MRC was not different from mean MRC on preoperative day $(P=0.43)$

On preoperative day, at the first postoperative day, at ICU discharge and at hospital discharge there were no differences in mean MRC between the NMES and control group $(P=0.92)$

MRC Medical Research Council, Cl confidence interval, ICU intensive care unit, NMES neuromuscular electrical stimulation

aAccording to the MRC scale [33], mean MRC score ranges from a minimum of 0 to a maximum of 5 points 
strength at hospital discharge compared to preoperative day $(P=0.008)$ (Table 6). The NMES intervention had no significant effect on grip strength (Table 6).

\section{Functional outcomes}

The secondary functional outcomes were evaluated for patients seen both on preoperative day and at hospital discharge $(n=12)$. The average mobility level and FIM score significantly decreased from preoperative day to hospital discharge (Table 7). On the other hand, the Timed Up and Go Test as well as the mental component score (MCS-12) and physical component score (PCS-12) of the SF-12 regained preoperative levels at hospital discharge (Table 7). Changes in all functional outcomes from preoperative day to ICU or hospital discharge were not significantly different between both groups.

\section{Patient satisfaction}

Forty-two patients were asked about their satisfaction with the NMES/sham stimulation. Out of 42 patients, 12 patients in the NMES group and 5 patients in the control group had a comfortable sensation $(P=0.03)$. Five patients in the NMES group and no patient in the control group had a feeling of discomfort $(P=0.048)$.

\section{Discussion}

In this randomized controlled trial, NMES had no effect on MLT, but was associated with a higher rate in regaining muscle strength during the ICU stay. At hospital discharge, all patients regained preoperative levels of muscle strength evaluated with the MRC scale, but still showed residual functional disability and decreased MLT compared to pre-ICU levels.

\section{Muscle layer thickness (MLT)}

The increase in MLT from preoperative day to postoperative day 1 can be attributed to the surgically induced inflammation and to the positive intraoperative fluid

Table 6 Linear mixed model for grip strength measured by hand dynamometer on four important study days (49 patients, 127 observations)

\begin{tabular}{lll}
\hline & $\begin{array}{l}\text { Estimate of mean MRC } \\
\text { in kilogram-force }(\mathrm{kgf})(95 \% \mathrm{Cl})\end{array}$ & $P$ value \\
\hline Intercept & $30.18(25.63$ to 34.73$)$ & $<0.001$ \\
Preoperative day & Reference & $\cdot$ \\
First postoperative day & $-11.55(-13.98$ to -9.11$)$ & $<0.001$ \\
ICU discharge & $-8.00(-10.47$ to -5.52$)$ & $<0.001$ \\
Hospital discharge & $-4.00(-6.90$ to -1.09$)$ & 0.008 \\
Control group & Reference & $\cdot$ \\
NMES group & $0.89(-5.16$ to 6.94) & 0.77 \\
\hline
\end{tabular}

MRC Medical Research Council, Cl confidence interval, ICU intensive care unit, NMES neuromuscular electrical stimulation balance. Fluid is entering the muscle, since cumulative fluid balance and change in MLT positively correlate in the first three postoperative days. Postoperatively, MLT progressively decreased until ICU discharge, but was still higher at ICU discharge than on preoperative day. This implies that the intramuscular edema regressed, but was still present at ICU discharge. Only at hospital discharge, MLT was lower than on preoperative day. This clearly indicates that also MLT was lost. It is not possible to tell at what time muscle loss started, since measurement of MLT was highly affected by intramuscular edema.

In the Catastim 2 study, NMES had no effect on MLT. Edema could have led to dissipation of the electrical current so that the current reaching the intramuscular motor nerve branches was not sufficient to generate muscle contractions. Segers [38] demonstrated that patients with high edema had insufficient muscle contractions during NMES. Segers found that patients had more sufficient NMES muscle contractions at the beginning of their ICU stay than after 1 week of ICU stay. However they did not analyze whether this was attributed to changes in development of edema or sepsis. In our study the positive correlation between cumulative fluid balance and change in MLT during the first three postoperative days suggests that edema is predominant in the early stage of surgical critical illness. Hence the effects of NMES may be rather reduced in the first days of critical illness. In the first ICU week, up to $62 \%$ of all patients had a nonexcitable muscle membrane [39], which may also attenuate the effects of NMES.

So far, only three studies $[9,19,40]$ showed an effect of NMES on muscle mass in critically ill patients. However variation in muscle mass was not compared to preICU values and fluid status was not considered as a confounder for measurement of muscle mass in these studies $[9,19,40]$. The Catastim 2 study indicates that the postoperative decrease in MLT at the ICU is not only due to loss of muscle mass, but also to reduction of intramuscular edema.

\section{Muscle strength}

The decrease in MRC strength from preoperative day to postoperative day 1 is most likely due to the surgical event and the postoperative sedation. Even before the NMES intervention began, the decrease in MRC from preoperative day to postoperative day 1 was coincidentally more pronounced in patients randomized to the NMES intervention than in patients randomized to the control group. After the onset of the NMES intervention, patients in the NMES group had a 4.5 times higher slope in recovering muscle strength during the ICU stay than patients in the control group. This indicates that the NMES intervention promotes a faster regain of muscle strength during the ICU stay. Still, both groups 
Table 7 Functional outcomes on four important study days (12 patients)

\begin{tabular}{|c|c|c|c|c|}
\hline & Preoperative day & ICU discharge & Hospital discharge & $\begin{array}{l}P \text { for change from preoperative } \\
\text { day to hospital discharge }\end{array}$ \\
\hline Average mobility level, median (range) & $12(12-12)$ & $2(0-11)^{a}$ & $12(8-12)^{b}$ & 0.04 \\
\hline FIM score, median (range) & $125.0(119-126)$ & & $122.0(82-126)$ & 0.02 \\
\hline Timed Up and Go Test, median (range) & $11.5(7-20)$ & & $11.5(1-28)$ & 0.24 \\
\hline MCS-12, median (range) & $52.82(25.05-64.63)$ & & $51.91(29.42-64.08)$ & 0.88 \\
\hline PCS-12, median (range) & $40.02(17.91-61.61)$ & & $32.58(27.46-45.90)$ & 0.53 \\
\hline
\end{tabular}

ICU intensive care unit, FIM Functional Independence Measure, MCS-12 mental component score of the SF-12, PCS-12 physical component score of the SF-12

${ }^{\mathrm{a}} P$ for change from preoperative day to ICU discharge: 0.002

${ }^{\mathrm{b}} \mathrm{P}$ for change from ICU discharge to hospital discharge: 0.002

were able to regain preoperative MRC values at hospital discharge. Nevertheless, a faster recovery may be beneficial and is an interesting result. Moreover, it shows that it is important to consider the factor time in the analysis of time variation of muscle strength in the ICU. No previous study $[16-18,21,22]$ yet accounted for an interaction between NMES and observation day in a linear regression model.

Similar to our results, Kho's study [16] showed a higher increase in MRC from ICU awakening to ICU and hospital discharges in the NMES group. In Kho's study, the absolute MRC values at hospital discharge were not different between both groups. Unfortunately, Kho did not assess MRC prior to the ICU in order to determine whether both groups could regain their preoperative muscle strength levels. Other studies also showed positive effects of NMES on the MRC score in ICU patients $[16-18,20-$ 22]. In line with these previous studies, the Catastim 2 study shows that NMES contributes to a faster recovery of muscle strength during the ICU stay.

\section{Functional outcomes}

In contrast to the recovery of muscle strength, patients did not regain their preoperative levels of mobility (average mobility level) and functional abilities (FIM score) at hospital discharge, independent of group allocation. This indicates that muscle strength and mobility may not be similarly affected by ICUAW. Similar to our results, Kho et al. [16] showed that the preoperative functional status score for the intensive care (FSS-ICU) score, which is similar to the FIM score, was not regained at hospital discharge, independent of group assignment. These functional disabilities at hospital discharge still persist 2 months to 5 years later as other trials have shown. After hospital discharge patients may regain their muscle strength, but not their functional abilities: patients with lower muscle strength at hospital discharge regained strength within 12 months [41]. However patients with lower muscle strength at hospital discharge still had lower SF-36 physical component score and lower 6-minute walking distance (6MWD) 3, 6, 12 and 24 months after ICU discharge [41]. Similarly, ICU survivors spent only
$3 \%$ of their time walking and $90 \%$ of their time inactive at 2 months after ICU discharge [42]. Their 6MWD was also decreased [42]. Likewise, Wieske [43] showed that patients with lower muscle strength during their ICU stay had lower SF-36 physical functioning 6 months after ICU discharge. Needham [44] also showed that acute lung injury survivors had lower 6MWD and SF-36 at 6 and 12 months after hospital discharge in comparison to normal predicted values. Even at 5 years after ICU discharge, 6MWD and SF-36 were reduced [3]. These impressive results clearly show that ICUAW is a public health issue going beyond hospital discharge, which calls for prophylactic or therapeutic measures. In our study, NMES had no effect on functional outcomes at hospital discharge. Yet, our sensitivity analysis for the secondary functional outcomes only included a small patient subcategory $(n=12)$ seen both on preoperative day and at hospital discharge. The sensitivity analysis was chosen in order to determine whether preoperative functional levels could be regained at hospital discharge. Positive effects of early mobilization on functional outcomes were shown in other studies: Schweickert [15] showed that sedation interruptions and physical therapy led to better functional outcomes at hospital discharge. Abdellaoui [18] found an increase in 6MWD in the NMES group. Zanotti [22] observed that NMES reduced the number of days needed to transfer from bed to chair. Parry [45] showed that combined electrical stimulation and cycling in critically ill patients led to a shorter delirium period.

\section{Limitations}

Even before the NMES intervention started, patients randomized to the NMES group had a higher decrease in MRC score than patients randomized to the control group. This higher decrease in MRC at the first postoperative day may also contribute to a higher rate in recovering MRC in the NMES group during the ICU stay. Hence, the higher rate in recovering MRC during the ICU stay may not be due to the NMES intervention alone, but also partly to the coincidentally higher decrease in MRC from preoperative day to the first postoperative day in the NMES group. 
The average mobility level was initially suggested by Brown in 2004 in the Journal of the American Geriatrics Society [37]. The average mobility level proposed by Brown is not mentioned among measurement instruments for impairments in the critically ill patient in Parry's systematic review [46]. There may be better functional scores to assess mobility. On the other hand, the FIM score is recommended in Parry's systematic review [46].

\section{Outlook}

Visualizing or palpating the NMES contractions was hardly possible in patients with high fluid retention in our study and in Seger's study [38]. Therefore it should be verified by ultrasonography whether muscles are contracting sufficiently during NMES in the presence of edema.

The assessment of baseline values prior to surgery or acute illness is of paramount importance in order to adjust for confounders related to surgery such as inflammation, fluid balance and sedation. Fluid balance should be taken into account when MLT is assessed by ultrasonography. Due to the lack of combined assessment of preoperative muscle mass values and fluid balance in previous studies, the formation and regression of intramuscular edema have never been shown before and need to be thoroughly addressed in future.

In line with previous studies [41], it is not the loss in muscle strength or mass but primarily the loss in functional outcomes which may play the more significant and patient-centered public health issue. Future studies should especially study patient-centered functional outcomes such as FIM, mobility and health-related quality of life (HRQoL).

\section{Conclusions}

NMES had no effect on MLT, but was associated with a higher rate in regaining muscle strength during the ICU stay. Regression of intramuscular edema during the ICU stay interfered with measurement of changes in MLT. At hospital discharge, all patients had regained preoperative levels of muscle strength evaluated with the MRC scale, but still showed residual functional disability and decreased MLT compared to pre-ICU levels.

\section{Key messages}

- The NMES intervention had no effect on muscle layer thickness, but was associated with a higher rate in regaining muscle strength during the ICU stay.

- Patients regained preoperative levels of muscle strength evaluated with the MRC scale by hospital discharge, but still had residual functional disability. This was independent of group allocation.

- The postoperative decrease in muscle layer thickness is not only due to loss of muscle mass, but also to reduction of intramuscular edema.

\section{Additional files}

\begin{abstract}
Additional file 1: Ultrasound scan of the left thigh at the lateral measuring point in the transverse and sagittal plane (Patient no. 104, control group) on postoperative day 3. (TIF $798 \mathrm{~kb}$ )
\end{abstract}

Additional file 2: Time variation of MLT and mean MRC of all muscle groups in individual patients. Days of ICU and hospital discharge are included. (PDF $837 \mathrm{~kb}$ )

Additional file 3: Linear mixed models for quadriceps mean MRC $^{\mathrm{a}}$ Table S1A. Linear mixed model for quadriceps mean MRC from the first postoperative day for a maximum of 14 postoperative days (51 patients, 219 observations) Days of ICU and hospital discharge, where no NMES was applied anymore, were excluded in this model. Table S1B. Linear mixed model for quadriceps mean MRC on four important study days (51 patients, 130 observations). (PDF $89.3 \mathrm{~kb}$ )

Additional file 4: Ultrasound scan of the left thigh at the medial measuring point in the transverse plane (Patient no. 84, control group). MLT was not evaluated on postoperative day 1. (TIF $1045 \mathrm{~kb}$ )

\section{Abbreviations}

6MWD: 6-Minute walking distance; Cl: Confidence interval; FIM: Functional Independence Measure; FSS-ICU: Functional status score for the intensive care unit; HRQoL: Health-related quality of life; ICC: Intra-class correlation coefficient; ICUAW: Intensive care unit-acquired weakness; MCS-12: Mental component score of the SF-12; MLT: Muscle layer thickness; MRC: Medical Research Council; NMES: Neuromuscular electrical stimulation; PCS-

12: Physical component score of the SF-12; SAPS II: Simplified Acute Physiology Score II; SD: Standard deviation; SF-12: 12-Item Short Form health survey; SF-36: 36-Item Short Form health survey.

\section{Competing interests}

The authors declare that they have no competing interests.

\section{Authors' contributions}

AF, MS, KA, AW, ASa, EMS, ASc, TPS and MH were responsible for study concept and design. AF, MS, KA, AW, ASa, MTH, MM and MH were responsible for acquisition, analysis or interpretation of data. AF drafted the manuscript. AF, MTH, MH and AW performed the statistical analysis. MM, ASC, EMS, MH and TPS provided administrative, technical or material support. $\mathrm{MH}$ supervised the study. All authors critically revised the manuscript for important intellectual content and approved the final version for submission. All authors agree to be accountable for all aspects of the work and have read and approved the final manuscript.

\section{Acknowledgments}

The study was supported by a research grant from the Medical University of Vienna. The funding sources had no role in design, in the collection, analysis, and interpretation of data; in the writing of the manuscript; and in the decision to submit the manuscript for publication. We thank the staff of the participating intensive care units, patients, relatives and caregivers who made this trial possible. None of these individuals received financial compensation for their contribution to this study.

\section{Author details}

${ }^{1}$ Division of Cardiothoracic and Vascular Anesthesia and Intensive Care Medicine, Department of Anesthesia, General Intensive Care and Pain Control, Medical University of Vienna, Währingergürtel 18-20, 1090 Vienna, Austria. ${ }^{2}$ Kantonsspital Sankt Gallen, Rorschacherstrasse 95, 9007 St. Gallen, Switzerland. ${ }^{3}$ Krankenhaus der Barmherzigen Schwestern Ried, Schlossberg 1, 4910 Ried im Innkreis, Austria. ${ }^{4}$ University Hospital Landeskrankenhaus Salzburg, Müllner Hauptstraße 48, 5020 Salzburg, Austria. ${ }^{5}$ Medical University of Vienna, Spitalgasse 23, 1090 Vienna, Austria. ${ }^{6}$ Center for Medical Statistics, Informatics and Intelligent Systems, Medical University of Vienna, Spitalgasse 23, 1090 Vienna, Austria. ${ }^{7}$ Institute for Physical Medicine and Rehabilitation, Kaiser-Franz-Josef-Spital Wien, Kundratstrasse 3, 1100 Vienna, Austria. ${ }^{8}$ Institute for Physical Medicine and Rehabilitation, Donauspital Wien, Langobardenstraße 122, 1220 Vienna, Austria. 
Received: 3 October 2015 Accepted: 18 January 2016 Published online: 29 January 2016

\section{References}

1. Latronico N, Bolton CF. Critical illness polyneuropathy and myopathy: a major cause of muscle weakness and paralysis. Lancet Neurol. 2011;10:931-41. doi:10.1016/S1474-4422(11)70178-8.

2. Mirzakhani H, Williams JN, Mello J, Joseph S, Meyer MJ, Waak K, et al. Muscle weakness predicts pharyngeal dysfunction and symptomatic aspiration in long-term ventilated patients. Anesthesiology. 2013;119:389-97. doi:10.1097/ ALN.0b013e31829373fe.

3. Herridge MS, Tansey CM, Matte A, Tomlinson G, Diaz-Granados N, Cooper A, et al. Functional disability 5 years after acute respiratory distress syndrome. N Engl J Med. 2011;364:1293-304. doi:10.1056/NEJMoa1011802.

4. Iwashyna TJ, Ely EW, Smith DM, Langa KM. Long-term cognitive impairment and functional disability among survivors of severe sepsis. JAMA. 2010;304:1787-94. doi:10.1001/jama.2010.1553.

5. Stevens RD, Marshall SA, Cornblath DR, Hoke A, Needham DM, de Jonghe B, et al. A framework for diagnosing and classifying intensive care unitacquired weakness. Crit Care Med. 2009;37:S299-308. doi:10.1097/CCM. ob013e3181b6ef67.

6. Cartwright MS, Kwayisi G, Griffin LP, Sarwal A, Walker FO, Harris JM, et al. Quantitative neuromuscular ultrasound in the intensive care unit. Muscle Nerve. 2013;47:255-9.

7. Puthucheary ZA, Rawal J, McPhail M, Connolly B, Ratnayake G, Chan P, et al. Acute skeletal muscle wasting in critical illness. JAMA. 2013;310:1591-600. doi:10.1001/jama.2013.278481.

8. Campbell IT, Watt T, Withers D, England R, Sukumar S, Keegan MA, et al. Muscle thickness, measured with ultrasound, may be an indicator of lean tissue wasting in multiple organ failure in the presence of edema. Am J Clin Nutr. 1995;62:533-9.

9. Gerovasili V, Stefanidis K, Vitzilaios K, Karatzanos E, Politis P, Koroneos A, et al. Electrical muscle stimulation preserves the muscle mass of critically ill patients: a randomized study. Crit Care. 2009;13:R161. doi:10.1186/cc8123.

10. Moukas M, Vassiliou MP, Amygdalou A, Mandragos C, Takis F, Behrakis PK. Muscular mass assessed by ultrasonography after administration of lowdose corticosteroids and muscle relaxants in critically ill hemiplegic patients. Clin Nutr. 2002;21:297-302.

11. Poulsen JB, Moller K, Jensen CV, Weisdorf S, Kehlet H, Perner A. Effect of transcutaneous electrical muscle stimulation on muscle volume in patients with septic shock. Crit Care Med. 2011;39:456-61. doi:10.1097/CCM. Ob013e318205c7bc.

12. Puthucheary ZA, Phadke R, Rawal J, McPhail MJ, Sidhu PS, Rowlerson A, et al. Qualitative ultrasound in acute critical illness muscle wasting. Crit Care Med. 2015;43:1603-11. doi:10.1097/CCM.0000000000001016.

13. Reid CL, Campbell IT, Little RA. Muscle wasting and energy balance in critical illness. Clin Nutr. 2004;23:273-80. doi:10.1016/S0261-5614(03)00129-8.

14. Hermans $G$, De Jonghe B, Bruyninckx F, Van den Berghe $G$. Interventions for preventing critical illness polyneuropathy and critical illness myopathy. Cochrane Database Syst Rev. 2014;1:CD006832. doi:10.1002/14651858. CD006832.pub3.

15. Schweickert WD, Pohlman MC, Pohlman AS, Nigos C, Pawlik AJ, Esbrook CL, et al. Early physical and occupational therapy in mechanically ventilated, critically ill patients: a randomised controlled trial. Lancet. 2009;373:1874-82. doi:10.1016/S0140-6736(09)60658-9.

16. Kho ME, Truong AD, Zanni JM, Ciesla ND, Brower RG, Palmer JB, et al. Neuromuscular electrical stimulation in mechanically ventilated patients: a randomized, sham-controlled pilot trial with blinded outcome assessment. J Crit Care. 2015;30:32-9. doi:10.1016/j.jcrc.2014.09.014.

17. Rodriguez PO, Setten M, Maskin LP, Bonelli I, Vidomlansky SR, Attie S, et al. Muscle weakness in septic patients requiring mechanical ventilation: protective effect of transcutaneous neuromuscular electrical stimulation. J Crit Care. 2012;27:319. doi:10.1016/j.jcrc.2011.04.010. e1-8.

18. Abdellaoui A, Prefaut C, Gouzi F, Couillard A, Coisy-Quivy M, Hugon G, et al. Skeletal muscle effects of electrostimulation after COPD exacerbation: a pilot study. Eur Respir J. 2011;38:781-8. doi:10.1183/09031936.00167110.

19. Gruther W, Kainberger F, Fialka-Moser V, Paternostro-Sluga T, Quittan M, Spiss C, et al. Effects of neuromuscular electrical stimulation on muscle layer thickness of knee extensor muscles in intensive care unit patients: a pilot study. J Rehabil Med. 2010;42:593-7. doi:10.2340/16501977-0564.
20. Karatzanos E, Gerovasili V, Zervakis D, Tripodaki ES, Apostolou K, Vasileiadis I, et al. Electrical muscle stimulation: an effective form of exercise and early mobilization to preserve muscle strength in critically ill patients. Crit Care Res Pract. 2012;2012:432752. doi:10.1155/2012/432752

21. Routsi C, Gerovasili V, Vasileiadis I, Karatzanos E, Pitsolis T, Tripodaki E, et al. Electrical muscle stimulation prevents critical illness polyneuromyopathy: a randomized parallel intervention trial. Crit Care. 2010;14:R74. doi:10.1186/cc8987.

22. Zanotti E, Felicetti G, Maini M, Fracchia C. Peripheral muscle strength training in bed-bound patients with COPD receiving mechanical ventilation: effect of electrical stimulation. Chest. 2003;124:292-6.

23. Iwatsu K, Yamada S, lida Y, Sampei H, Kobayashi K, Kainuma M, et al. Feasibility of neuromuscular electrical stimulation immediately after cardiovascular surgery. Arch Phys Med Rehabil. 2015;96:63-8. doi:10.1016/j. apmr.2014.08.012.

24. Janda D. Effects of early neuromuscular electrical stimulation on the protein catabolism in critically ill patients. Diploma thesis, Medical University of Vienna; 2010. German

25. Lozano R, Naghavi M, Foreman K, Lim S, Shibuya K, Aboyans V, et al. Global and regional mortality from 235 causes of death for 20 age groups in 1990 and 2010: a systematic analysis for the Global Burden of Disease Study 2010. Lancet. 2013:380:2095-128.

26. Errath M, Berghold A, Ofner P, Quehenberger F. Randomizer for clinical trials 1.8.1. https://www.meduniwien.ac.at/randomizer/web/login.php. Accessed 1 November 2015.

27. Parry SM, Berney S, Koopman R, Bryant A, El-Ansary D, Puthucheary Z, et al. Early rehabilitation in critical care (eRiCC): functional electrical stimulation with cycling protocol for a randomised controlled trial. BMJ Open. 2012;2:e001891. doi:10.1136/bmjopen-2012-001891.

28. Seymour JM, Ward K, Sidhu PS, Puthucheary Z, Steier J, Jolley CJ, et al. Ultrasound measurement of rectus femoris cross-sectional area and the relationship with quadriceps strength in COPD. Thorax. 2009;64:418-23. doi:10.1136/thx.2008.103986.

29. Trappe TA, Lindquist DM, Carrithers JA. Muscle-specific atrophy of the quadriceps femoris with aging. J Appl Physiol. 2001;90:2070-4.

30. Narici MV, Hoppeler H, Kayser B, Landoni L, Claassen H, Gavardi C, et al. Human quadriceps cross-sectional area, torque and neural activation during 6 months strength training. Acta Physiol Scand. 1996;157:175-86.

31. Strasser EM, Draskovits T, Praschak M, Quittan M, Graf A. Association between ultrasound measurements of muscle thickness, pennation angle, echogenicity and skeletal muscle strength in the elderly. Age (Dordr). 2013;35:2377-88. doi:10.1007/s11357-013-9517-z

32. Vanpee G, Hermans G, Segers J, Gosselink R. Assessment of limb muscle strength in critically ill patients: a systematic review. Crit Care Med. 2014:42:701-11. doi:10.1097/CCM.0000000000000030.

33. Van der Ploeg $\mathrm{R}$, Oosterhuis $\mathrm{H}$, Reuvekamp J. Measuring muscle strength. J Neurol. 1984:231:200-3.

34. Hamilton BB, Laughlin JA, Fiedler RC, Granger CV. Interrater reliability of the 7-level functional independence measure (FIM). Scand J Rehabil Med. 1994;26:115-9.

35. Podsiadlo D, Richardson S. The timed "Up \& Go": a test of basic functional mobility for frail elderly persons. J Am Geriatr Soc. 1991;39:142-8.

36. Ware Jr J, Kosinski M, Keller SD. A 12-Item Short-Form Health Survey: construction of scales and preliminary tests of reliability and validity. Med Care. 1996:34:220-33.

37. Brown CJ, Friedkin RJ, Inouye SK. Prevalence and outcomes of low mobility in hospitalized older patients. J Am Geriatr Soc. 2004;52:1263-70. doi:10.1111/j.1532-5415.2004.52354.x.

38. Segers J, Hermans G, Bruyninckx F, Meyfroidt G, Langer D, Gosselink R. Feasibility of neuromuscular electrical stimulation in critically ill patients. J Crit Care. 2014;29:1082-8.

39. Bierbrauer J, Koch S, Olbricht C, Hamati J, Lodka D, Schneider J, et al. Early type II fiber atrophy in intensive care unit patients with nonexcitable muscle membrane. Crit Care Med. 2012:40:647-50.

40. Hirose T, Shiozaki T, Shimizu K, Mouri T, Noguchi K, Ohnishi M, et al. The effect of electrical muscle stimulation on the prevention of disuse muscle atrophy in patients with consciousness disturbance in the intensive care unit. J Crit Care. 2013;28:536. doi:10.1016/j.jcrc.2013.02.010. e1-7.

41. Fan E, Dowdy DW, Colantuoni E, Mendez-Tellez PA, Sevransky JE, Shanholtz C, et al. Physical complications in acute lung injury survivors: a 2-year longitudinal prospective study. Crit Care Med. 2014;42:849-59. 
42. Denehy L, Berney S, Whitburn L, Edbrooke L. Quantifying physical activity levels of survivors of intensive care: a prospective observational study. Phys Ther. 2012;92:1507-17.

43. Wieske L, Dettling-Ihnenfeldt DS, Verhamme C, Nollet F, van Schaik IN Schultz MJ, et al. Impact of ICU-acquired weakness on post-ICU physical functioning: a follow-up study. Crit Care. 2015;19:196.

44. Needham DM, Wozniak AW, Hough CL, Morris PE, Dinglas VD, Jackson JC, et al. Risk factors for physical impairment after acute lung injury in a national, multicenter study. Am J Respir Crit Care Med. 2014;189:1214-24.

45. Parry SM, Berney S, Warrillow S, El-Ansary D, Bryant AL, Hart N, et al. Functional electrical stimulation with cycling in the critically ill: a pilot case-matched control study. I Crit Care. 2014;29:695. e1-e7.

46. Parry SM, Granger $\mathrm{CL}$, Berney S, Jones J, Beach L, El-Ansary D, et al. Assessment of impairment and activity limitations in the critically ill: A systematic review of measurement instruments and their clinimetric properties. Intensive Care Med. 2015;41:744-62.

Submit your next manuscript to BioMed Central and we will help you at every step:

- We accept pre-submission inquiries

- Our selector tool helps you to find the most relevant journal

- We provide round the clock customer support

- Convenient online submission

- Thorough peer review

- Inclusion in PubMed and all major indexing services

- Maximum visibility for your research

Submit your manuscript at www.biomedcentral.com/submit
Biomed Central 